\title{
Docentes agradecidos y con bienestar: efecto de una intervención psicoeducativa*13
}

\author{
Diego García Álvarez \\ Doctorado en Ciencias de la Educación \\ Universidad Privada Rafael Belloso \\ Chacín. Maracaibo, Venezuela \\ Correo electrónico: diegogarcia_123@hotmail.com
}

María José Soler

Doctora en Psicología Universidad de Flores, Argentina Correo electrónico: mariajsoler@gmail.com
Recibido: 01/08/2019

Evaluado: 04/10/2019

Aceptado: 16/10/2019

\section{Resumen}

La literatura científica ha puesto de manifiesto la eficacia de las intervenciones basadas en la psicología positiva. Desde ese lugar, el objetivo de esta investigación es determinar la eficacia de una intervención psicoeducativa basada en psicología positiva aplicada al contexto educacional orientada a la promoción de la salud mental, bienestar psicológico y gratitud. Metodológicamente, el estudio se enmarca en un diseño cuasi experimental correlacional, un solo grupo de medias pre y post, en una muestra de 39 docentes, la recolección de datos fue a través de un cuadernillo comprendido por escalas para medir las variables de bienestar psicológico, gratitud y pesimismo, con adecuados índices de confiabilidad y validez. Los resultados muestran diferencias estadísticamente significativas del bienestar psicológico y gratitud, correlaciones positivas entre la gratitud y el bienestar psicológico, e inversamente con pesimismo. Se concluye que la intervención psicoeducativa puede ser una herramienta para la promoción de la salud mental en docentes.

13 Para citar este artículo: García-Alvarez, D. y Soler, M. J. (2021). Docentes agradecidos y con bienestar: efecto de una intervención psicoeducativa. Informes Psicológicos, 21(1), 201-215 http://dx.doi.org/10.18566/infpsic.v21n1a13 Proyecto de investigación financiado. Asociación Civil Jóvenes Fuertes, Uruguay. 


\title{
Grateful teachers and with well-being: effect of a psychoeducational intervention
}

\begin{abstract}
Scientific literature has shown the efficacy of interventions based on positive psychology. From this point of view, the objective of this research is to determine the efficacy of a psychoeducational intervention based on positive psychology applied to the educational context and aimed at promoting mental health, psychological well-being and gratitude. Methodologically, the study is framed in a correlational quasi-experimental design, a single group of pre and post means, in a sample of 39 teachers. The data collection was made through a booklet comprised of scales to measure the variables of psychological well-being, gratitude and pessimism, with adequate reliability and validity indices. The results show statistically significant differences in psychological well-being and gratitude, positive correlations between gratitude and psychological well-being, and inversely with pessimism. It is concluded that psychoeducational intervention can be a tool for promoting mental health in teachers.
\end{abstract}

\section{Keywords}

Teachers, gratitude, psychological well-being, psychoeducational intervention.

\section{Professores gratos e com bem-estar: efeito de uma intervenção psicoeducacional}

\section{Resumo}

A literatura científica tem demonstrado a eficácia de intervenções baseadas na psicologia positiva. A partir daí 0 objetivo desta pesquisa é determinar a eficácia de uma intervenção psicoeducacional baseada na psicologia positiva aplicada ao contexto educacional que visa a promoção da saúde mental, bem-estar psicológico e gratidão. Metodologicamente, o estudo está enquadrado em um delineamento quase experimental correlacional, um único conjunto de médias antes e depois, em uma amostra de 39 professores, a coleta de dados se deu por meio de uma cartilha composta por escalas para mensurar as variáveis de bem-estar psicológico, gratidão e pessimismo, com índices adequados de confiabilidade e validade. 0 s resultados mostram diferenças estatisticamente significativas no bem-estar psicológico e gratidão, correlações positivas entre gratidão e bem-estar psicológico, e inversamente com pessimismo. Conclui-se que a intervenção psicoeducacional pode ser um instrumento de promoção da saúde mental em professores. 


\section{ntroducción}

El enfoque de la psicología positiva trajo consigo un novedoso constructo transversal en su modelo teórico denominado: virtudes y fortalezas del carácter propuesto por Peterson \& Seligman (2004). En ese sentido, la psicología positiva es un enfoque dentro de la ciencia psicológica que se encarga del estudio del bienestar, la experiencia óptima y las virtudes humanas. Para este enfoque las fortalezas del carácter son rasgos de la personalidad con valor moral, a través de los cuales se expresan operacionalmente las virtudes. Asimismo, los autores explican que las virtudes y fortalezas del carácter se relacionan, viabilizan o producen el bienestar psicológico de las personas mediante su identificación, concienciación y puesta en práctica voluntariosa en la vida cotidiana, generando lo que se denomina una buena vida en el estricto sentido aristotélico eudaimónico.

La psicología positiva como enfoque científico toma sus raíces en la eudaimonía en concebir el bienestar como producto de una vida bien llevada, es decir, mediante la utilización de las fortalezas del carácter para el bien individual y común. De ahí que los modelos teóricos que toman relevancia con esta visión son los modelos multidimensionales del bienestar psicológico, como por ejemplo PERMA o florecer de Seligman (2011) y el modelo basado en el crecimiento personal de Ryff (1989; 2014). El modelo multidimensional del bienestar propuesto por Ryff está basado en el crecimiento continuo del potencial humano explicado a través de seis dimensiones interconectadas entre sí: aceptación, vínculos, autonomía, proyectos, dominio del ambiente que van funcionando de manera óptima posibilitando la dimensión del crecimiento personal de manera duradera a lo largo del ciclo vital. En consideración de Peterson \& Seligman (2004), el bienestar psicológico va a ser configurado en la forma como la persona identifique, reconozca y ponga en práctica sus virtudes y fortalezas del carácter facilitando el funcionamiento psicológico pleno.

Siguiendo con la idea, las virtudes humanas han sido clasificadas por Peterson \& Seligman (2004) en seis grupos con fortalezas del carácter correspondientes en las siguientes categorías: sabiduría, coraje, templanza, civismo, humanidad y trascendencia. La virtud de la trascendencia hace referencia al grupo de fortalezas que permiten a la persona conectar con la inmensidad del universo, cultivar el sentido de la vida y se denominan de esa forma debido a que viabilizan procesos de vinculación con lo que nos rodea permitiendo trascender de la individualidad. Las fortalezas que nos permiten conectarnos son: apreciación a la belleza, optimismo y esperanza, humor, espiritualidad y la gratitud.

Según el diseño de investigación, se va a profundizar sobre la fortaleza de la gratitud. Peterson \& Seligman (2004) refieren que es una fortaleza relacionada con componentes cognitivos y emocionales, explicando que la fortaleza de la gratitud es un sentir de agradecimiento y alegría en respuesta a recibir algún regalo, por un beneficio, sea o no tangible, o por un momento de felicidad evocable. De modo que la fortaleza de la gratitud consiste en ser consciente de las cosas buenas que suceden en la vida, emocionarse, dar 
gracias por esas cosas y dedicar tiempo a agradecerlo; nótense los componentes cognitivos, conductuales y afectivos del constructo.

Ahora bien, son los autores Emmons \& McCullough (2003) quienes han profundizado sobre el estudio de la gratitud, señalando que se trata de un constructo complejo que abarca dimensiones de orden: a) cognitivo: en darse cuenta que se ha sido beneficiado, b) afectivo: en emocionarse por esa percepción generando alegría o el sentimiento de agradecimiento, y c) conductual: por expresar ese agradecimiento ya sea en decir gracias o mediante vías más elaboradas para tales fines. De igual forma, estos autores proponen estrategias para el desarrollo de la fortaleza de la gratitud en esferas terapéuticas o de intervención psicológica como el ejercicio de contar bendiciones y otros más publicados con adecuadas evidencias de sus efectos sobre el bienestar psicológico, correlatos emocionales e interpersonales.

A nivel de evidencias empíricas, Watkins, Uhder \& Pichinevskiy (2015) realizaron un estudio controlado con tres grupos para determinar la efectividad de la gratitud a modo de tratamiento para mejorar el bienestar subjetivo. El grupo con el tratamiento basado en las estrategias de gratitud superó en nivel significativo a los grupos de control en el aumento del bienestar; los autores sugieren que los programas sobre gratitud puede ser una intervención para mejorar el bienestar y educar los sesgos de procesamiento de información, de pensamiento y de comparación de forma más saludable, es decir, enseñar a la persona a centrarse en reconocer las llamadas bendiciones sobre las adversidades, o sobre lo que sí se tiene o no se tiene en la vida.
Por su parte, Witvliet, Richie, RootLuna \& Van-Tongeren (2019) han explicado a través de regresiones jerárquicas que la gratitud tiene poder predictivo sobre la esperanza y la felicidad de las personas, incluso mayor poder predictivo que otras fortalezas del carácter como el autocontrol, la modestia y el perdón. Además, Dickens (2017), en un meta análisis acerca de la efectividad de intervenciones de gratitud, ha encontrado que tiene numerosos correlatos beneficiosos haciendo énfasis en el bienestar y felicidad.

Se han reportado algunos antecedentes de investigaciones basados en intervenciones centradas en promover el bienestar y gratitud en docentes, a saber: Chan (2010) realizó un estudio con diseño pre y post en una muestra de 96 docentes chinos a través de un programa gratitud de ocho semanas, los resultados indicaron efectividad estadísticamente significativa en el incremento del afecto positivo y satisfacción con la vida, así como correlaciones significativas positivas entre satisfacción con la vida y gratitud e inversamente significativas con burnout.

Asimismo, Chan (2011) realizó otro estudio para probar la efectividad de un programa de gratitud para la promoción de la satisfacción con la vida y reducir síntomas del burnout con una muestra de 63 docentes chinos por medio de un método pre y post. Los resultados arrojaron cambios significativos, es decir, hubo aumento de la satisfacción con la vida, así como reducción de algunos de los síntomas del burnout (agotamiento emocional) en interacción con orientación a la felicidad. El autor refiere la importancia de incluir temas relacionados con los aspectos positivos de las personas y la felicidad en la 
vida en programas orientados a promover la salud mental y bienestar en docentes para impedir el burnout.

En el 2013, Chan presentó un estudio basado en comparar los enfoques de intervención centrados en la gratitud y en estrategias de afrontamiento para la promoción del bienestar subjetivo en una muestra de 78 maestros chinos. La investigación encontró que en ambos grupos se aumentó la satisfacción con la vida, es decir, el grupo con la intervención basada en las estrategias de afrontamiento y el grupo con la intervención basada en gratitud, pero en este último grupo se encontraron diferencias estadísticamente significativas en el aumento de la satisfacción con la vida y experiencias emocionales relacionadas con el conteo de eventos positivos o llamados por el autor: contando las bendiciones de la vida.

La literatura científica en el contexto Iberoamericano refiere el antecedente del estudio realizado por Martínez-Martí, Avia y Hernández-Lloreda (2010) con el objetivo de examinar los efectos de la intervención de gratitud denominada contando bendiciones, propuesta por Emmons y McCullough (2003) en una muestra española. El estudio fue un ensayo controlado a través de tres grupos formados de manera aleatoria. Los resultados sugieren que las intervenciones en gratitud pueden ser promotoras del bienestar en los participantes.

De igual manera, antecedentes han puesto de manifiesto la efectividad de las intervenciones basadas en psicología positiva centradas en la identificación, reconocimiento y puesta en práctica consciente de las fortalezas del carácter en la promoción del bienestar psicológico
(Buschor, Proyer, \& Ruch, 2013; Lavy, 2019; Proyer, Ruch, \& Buschor, 2012; Wellenzohn, Proyer, \& Ruch, 2016). Por su parte, Sin \& Lyubomirsky (2009) refieren que la efectividad de las intervenciones basadas en psicología positiva consiste en que la persona reconoce múltiples recursos que puede implementar en el desarrollo de su potencial, así como en el entrenamiento de estilos explicativos de la realidad que permitirían la re-estructuración cognitiva de la misma fortaleciendo la tendencia al manejo de expectativas reales para una evaluación más adaptativa con fines de mantenimiento del bienestar.

De ahí que se entienden como estilos explicativos de la realidad aquellas expectativas generalizadas favorables o desfavorables sobre objetivos, sucesos y cosas que sucedan en la vida, es decir, el optimismo sería esa expectativa de éxito sobre el fracaso, mientras que el pesimismo sería lo contrario (Ferrando, Chico \& Tous, 2002). Se puede pensar que luego de entrenamientos basados en psicología positiva con fundamento cognitivo conductual mediante la práctica de las fortalezas del carácter se puedan modificar las expectativas a futuro disminuyendo el pesimismo, sugiriendo que, al entrenar o modificar los esquemas cognitivos, se afectan también los estilos explicativos / atribucionales de la realidad; antecedentes de investigación han puesto en evidencia los beneficios de intervenciones de psicología positiva sobre el optimismo y bienestar de los participantes (Reivich, Gillham, Chaplin \& Seligman, 2013).

En definitiva, los antecedentes descritos ponen de manifiesto la posible eficacia de intervenciones psicoeducativas basadas en psicología positiva en la promoción de la salud mental y de otros 
correlatos beneficiosos en sus participantes. Entonces, se plantea esta intervención diseñada bajo el enfoque de la psicología positiva aplicada a la educación con el fin de promover el bienestar psicológico y la fortaleza de la gratitud como una forma de cultivar la virtud de la transcendencia. Asimismo, la justificación teórica - práctica de esta investigación se trata de un antecedente científico metodológico sobre una intervención psicoeducativa orientada a la salud mental de docentes en contexto latinoamericano, esencialmente uruguayo, así como ser también un futuro referente teórico y de intervención para profesionales abocados a la salud ocupacional del docente. En resumen, este estudio busca aportar a la comunidad científica latinoamericana datos de intervención psicológica de solidez empírica con pertinencia en el contexto local.

De acuerdo a lo anteriormente expuesto, se plantean las siguientes preguntas orientadoras del problema: a) ¿cuál será el nivel de bienestar psicológico y gratitud en los participantes antes y después de la aplicación de la intervención?, b) ¿existirán diferencias en las medidas antes y después del bienestar psicológico y la gratitud en los docentes participantes de la intervención psicoeducativa?, y c) ¿cuál será la relación entre la gratitud con bienestar psicológico y el pesimismo en las medidas post intervención?

De modo que estas formulaciones se operacionalizan en los siguientes objetivos de investigación: a) Medir el bienestar psicológico y gratitud antes / después de la aplicación de la intervención psicoeducativa; b) Determinar si existen diferencias estadísticamente significativas entre las medidas pre y post intervención; y c) Establecer las relaciones entre las variables de gratitud con bienestar psicológico y pesimismo. Todos objetivos orientados a caracterizar la eficacia de una intervención psicoeducativa basada en psicología positiva aplicada al contexto educacional con el propósito de la promoción de la salud mental, bienestar psicológico y gratitud en docentes participantes.

Por consiguiente, el sistema de hipótesis que orienta este trabajo es: a) Aumentarán las medidas del bienestar y gratitud en docentes participantes luego de la intervención psicoeducativa; b) Existirán diferencias estadísticamente significativas entre las medidas pre y post; y c) Existirán correlaciones positivas entre la gratitud y el bienestar, así como negativas entre la gratitud y el pesimismo.

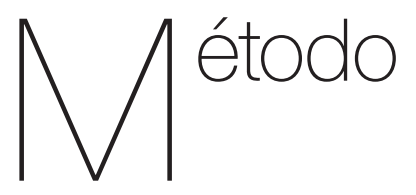

\section{Diseño}

El estudio se clasifica en un diseño cuasi experimental con un solo grupo con medidas pre y post de acuerdo a la guía metodológica de estudios en psicología de Montero y León (2007), así como en un diseño correlacional (Hernández, Fernández \& Baptista, 2008).

\section{Participantes}

La muestra del estudio estuvo compuesta por 39 docentes participantes de un curso de psicología positiva aplicada a la educación, y fue seleccionada a 
través de la fundación patrocinadora por medio de un llamado público dirigido a docentes de cualquier nivel y modalidad del sistema educativo uruguayo; el criterio de inclusión del llamado era interés por parte del docente en formarse en psicología positiva y estar en actual servicio o desempeño docente. En ese sentido, la muestra estuvo conformada por 6 participantes masculinos (16\%) y 33 participantes femeninas (84\%), y una media de edad de 36.9 años.

\section{Instrumentos de recolección de datos}

Se elaboró un cuadernillo con:

a) Carta de consentimiento informado y descripción de la investigación. Sección de datos socio - demográficos ad hoc.

b) Sub escala de la gratitud del Inventario de las Virtudes y Fortalezas del carácter propuesto por Peterson y Seligman (2004) validado por Rojas y Feldman (2010). Es un inventario conformado por una escala Likert para evaluar las virtudes y fortalezas del carácter, puede aplicarse en su totalidad o por sub escalas que midan las fortalezas. La sub escala de la gratitud está compuesta por 10 ítems, con opciones de respuesta del 1 al 5 que van desde muy en desacuerdo a muy de acuerdo; las autoras presentan evidencias de validez de contenido, técnicas de análisis factorial y consistencia interna de las escalas y cada una de sus subescalas. Específicamente, presenta adecuados índices de confiabilidad y validez, a saber: alfa de Cronbach .99 .

c) Escala de Bienestar Psicológico de Casullo (Bieps - A; Casullo, 2002). Se trata de una escala diseñada desde el modelo multidimensional de bienestar planteado por Ryff (1989; 2014). Evalúa el bienestar desde una perspectiva de crecimiento personal en sus dimensiones: proyectos, aceptación, control y vínculos. Casullo explica que la escala está conformada por 13 ítems con opciones de 1 al 3, y que su corrección debe hacerse sólo calculando el puntaje general de bienestar psicológico y realizar interpretaciones cualitativas de las cuatro dimensiones que lo componen, sugerencia realizada por la autora con base a los resultados de los análisis factoriales realizados en el estudio original. La escala presenta adecuadas propiedades psicométricas, a saber: alfa de Cronbach .75.

d) Sub escala de pesimismo del cuestionario Life Orientation Test (LOT - R; Scheier \& Carver, 1985) en la versión en español validada por Ferrando et al. (2002). Es una escala Likert conformada por 3 ítems que miden la disposición al pesimismo con opciones de respuesta del 1 al 5. Presenta propiedades psicométricas adecuadas que puede ser empleada para evaluar el pesimismo disposicional (alfa de Cronbach de .70).

\section{Procedimiento}

La investigación fue diseñada bajo los criterios éticos y legales expuestos por el Ejecutivo Uruguayo (2008) en el 
Decreto 379/008 y la Asociación Americana de Psicología (APA, 2010) para la investigación psicológica con adultos. Así pues, una vez esbozado el estudio, se procedió a la recolección de datos en la primera sesión de formación de la intervención. Los cuadernillos fueron suministrados por la docente facilitadora quien explicó el carácter voluntario, confidencial y de fines científicos del mismo. Vale destacar que cada cuadernillo constaba con el consentimiento informado, leído en voz alta por la docente y la invitación por escrito a participar. Al final de la intervención psicoeducativa, la docente facilitadora repitió el procedimiento descrito para la recolección de datos considerada medida post. Los datos pre y post fueron transcriptos, procesados y analizados luego del cierre de la experiencia formativa.

A nivel de la intervención psicoeducativa, la misma estuvo basada en la psicología positiva como eje teórico modulador buscando aplicaciones prácticas en el contexto educacional. En ese sentido, quedó constituida por un programa de ocho encuentros a modo de sesiones, a saber: 1) Introducción a la psicología positiva; 2) Educación del carácter, emocional y para el bienestar; 3) Vías a la felicidad y para el bienestar; 4) Resiliencia, estilos explicativos de la realidad: optimismo y pesimismo; 5) Virtudes y Fortalezas del Carácter; 6) Profundización en fortalezas del carácter: coraje y trascendencia; 7) Bienestar docente, empatía y vocación servicio; y 8) Presentación de proyectos relacionados a la aplicación de los postulados aprendidos en el contexto educacional. De igual manera, hubo un evento central que cada docente debía replicar en su centro educativo denominado: la semana de la gratitud.

La intervención psicoeducativa tuvo una duración de 56 horas de formación llevada a cabo los días sábados. A nivel de mediación didáctico andragógico, la intervención tenía el objetivo general de formar educadores capaces de aplicar psicología positiva en su contexto personal y educacional. A nivel de intervención psicológica tenía el objetivo de mantener o aumentar el bienestar psicológico y la gratitud en los participantes, es decir, la promoción de la salud mental en docentes.

Asimismo, las ocho sesiones de formación que constituían la intervención psicoeducativa estuvieron a cargo de dos docentes expertas en psicología positiva aplicada a la educación. La jornada de trabajo estuvo diseñada desde explicaciones magistrales, ejemplificaciones, estudio de estrategias prácticas de la psicología positiva para aplicación en campo personal y laboral educacional, actividades vivenciales que permitan el aprendizaje, así como la construcción transversal de un proyecto de aplicación de lo aprendido, que fue presentado al final de la intervención.

\section{Análisis de datos}

Los datos fueron transcriptos al procesador estadístico IBM SPSS Statistics 23.0. Primeramente, se realizaron análisis de estadísticas descriptivas enfatizados en medidas de tendencia central. Luego, se procedió a explorar la distribución de las variables mediante la prueba Shapiro - Wilk recomendada para muestras menores a 50 sujetos. Debido 
al resultado, se asumió la distribución no normal implicando decisiones prácticas que se materializaron en el uso de la estadística inferencial no paramétrica para la resolución de los objetivos planteados, a saber, las pruebas de rangos de Wilcoxon y el coeficiente de correlación Rho de Spearman. Para determinar el tamaño del efecto de la intervención sobre las variables de bienestar psicológico y gratitud, se empleó el programa G -Power, usando el método de estimación de la potencia y del tamaño del efecto basado en la eficiencia relativa asintótica aplicada a la prueba de Wilcoxon.

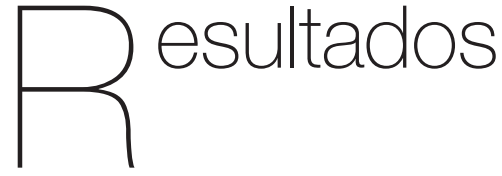

En la Tabla 1 se pueden apreciar los valores descriptivos de las variables de bienestar psicológico y la fortaleza de la gratitud tanto en la medida pre como en la medida post de la psicointervención. Se pueden apreciar incrementos en las medias de las variables de bienestar psicológico y gratitud tomando como referencia la medida pre a la medida post final de intervención.

Tabla 1

Descriptivos de las medidas pre y post

\begin{tabular}{lccccc}
\hline & N & Mínimo & Máximo & Media & $\begin{array}{c}\text { Desviación } \\
\text { estándar }\end{array}$ \\
\hline Pre Bienestar & 39 & 28.00 & 39.00 & 35.25 & 2.47 \\
Post Bienestar & 39 & 32.00 & 39.00 & 36.58 & 1.87 \\
Pre Gratitud & 39 & .00 & 50.00 & 42.02 & 8.19 \\
Post Gratitud & 39 & 11.00 & 50.00 & 44.41 & 6.52 \\
\hline
\end{tabular}

Luego de calcular los estadísticos descriptivos se procedió a realizar el análisis de contraste de normalidad a través de la prueba Shapiro - Wilk, debido al tamaño de la muestra inferior a 50 sujetos. Los valores de significancia fueron $<.05$ para cada una de las variables, por lo que se asumen distribuciones no normales. De modo que se manejaron análisis no paramétricos para seguir dando respuesta a los objetivos de investigación.

En ese sentido, para conocer la eficacia de la intervención psicoeducativa sobre las variables de bienestar psicológico y gratitud se empleó la prueba de los rangos con signos de Wilcoxon para muestras relacionadas. Los resultados muestran que hubo incremento en el bienestar psicológico $(z=-2.663, p<.05)$ y en la gratitud $(z=-3.686, p<.05)$, es decir, hubo incrementos estadísticamente significativos de las variables bienestar psicológico y gratitud tomando en consideración las medidas pre y post de los docentes participantes de la intervención. El tamaño del efecto de la intervención sobre el bienestar psicológico es pequeño llegando a moderado detectado con una potencia de .745. Con respecto al tamaño de la intervención sobre la variable gratitud, el efecto es moderado con una potencia detectada de .977 .

Asimismo, en las medidas post intervención se realizaron las pruebas de 
correlaciones a través del coeficiente de Rho de Spearman, teniendo los siguientes coeficientes positivos entre la gratitud con: bienestar psicológico $\left(r=.350^{*}\right)$, y sus dimensiones: aceptación $\left(r=.500^{\star *}\right)$, autonomía $(r=.178)$, vínculos $(r=.155)$ y proyectos $(r=.190)$, así como coeficiente negativo entre la gratitud y el pesimismo $(r=-.205)$.

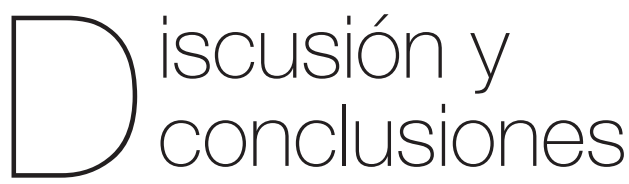

De acuerdo a los postulados de Peterson y Seligman (2004), se puede interpretar que la intervención psicoeducativa del presente estudio basada en psicología positiva aplicada al contexto educacional mostró adecuada evidencia de su eficacia en el aumento de la gratitud, es decir, la promoción de la fortaleza de la gratitud en los participantes puede ser entendida como el desarrollo de una percepción de ser beneficiado por hechos externos a la voluntad personal causando un sentimiento agradable, de alegría y, por supuesto, de agradecimiento, nótese los componentes cognitivos y emocionales del constructo. De modo que los participantes aumentan la consciencia sobre detalles concretos de su vida, ya sea personal o profesional, que generan el sentimiento de agradecimiento.

Asimismo, se interpreta con los postulados de Ryff (1989; 2014) y Casullo (2002) que la intervención psicoeducativa mostró adecuada evidencia en la promoción del bienestar psicológico en los participantes, es decir, desarrollaron las dimensiones interconectadas del bienestar psicológico multidimensional enfocado al crecimiento personal, a saber: sensación de control en situaciones, competencia para el manejo adecuado de contextos y ambientes, movilización de procesos de autoaceptación del sí mismo tanto en sus lados luminosos y los que no lo son tanto, es decir, la aceptación del pasado y del presente. Además, desarrollo de vínculos sociales sanos, planteamiento o concienciación de proyectos que le permiten cultivar el significado de la vida y, por supuesto, el desarrollo de la autonomía personal para comprender que se pueden tomar decisiones y tener la confianza en su buen juicio en la vida.

Los resultados expuestos son congruentes con los estudios anteriores que han puesto de manifiesto que las intervenciones en psicología positiva pueden ser herramientas eficaces para la promoción de la salud mental, bienestar y gratitud en sus participantes (Chan, 2010; Cunha, Pellanda \& Reppold, 2019; Dickens, 2017). Del mismo modo, la literatura ha puesto de manifiesto la relación inversa de la gratitud con características psicopatológicas (Deng et al., 2019; Jans-Beken, Lataster, Peels, Lechner \& Jacobs, 2018); en efecto, la fortaleza de la gratitud correlacionó inversamente con el pesimismo disposicional (Giménez, 2010).

En el presente estudio se encontraron correlaciones positivas entre la fortaleza de la gratitud y cada una de las dimensiones del bienestar psicológico. Peterson \& Seligman (2004) refieren que la gratitud es una fortaleza del carácter debido a que viabiliza el bienestar psicológico personal y social. Incluso otras investigaciones han mostrado que la fortaleza de la gratitud es una de las que más predice el bienestar 
psicológico y otros correlatos de salud mental en distintas etapas del ciclo vital (Buschor, Proyer, \& Ruch, 2013; CastroSolano \& Cosentino, 2016; Park \& Peterson, 2009; Peterson, Ruch, Beerman, Park \& Seligman, 2007; Porto-Noronha \& da Fonseca-Martins, 2016; Vela, Sparrow, Ikonomopoulos, González \& Rodríguez, 2017).

De alguna manera, la fortaleza de la gratitud cumple su rol dentro de la virtud de la trascendencia, debido a que logra que el sujeto traspase su realidad individual egocéntrica y factiblemente sobre - merecedora hacía una apertura de consciencia acerca de cosas, personas, situaciones y objetos que da por sentado en su existencia, comenzando a "contar bendiciones" como las denominan Emmons \& McCullough (2003); éstos refieren que el hecho de contar bendiciones permite a la persona entrenar su atención, pensamiento y estilo cognitivo en detectar aspectos de la vida para estar agradecidos.

Desde esta premisa cognitivo conductual en la compresión de la fortaleza de la gratitud a nivel de procesamiento cognitivo de la realidad, se puede sugerir que, al entrenar o modificar los esquemas cognitivos, se pueden también afectar los estilos explicativos / atribucionales de la realidad, es decir, aquellas expectativas generalizadas acerca de qué tan favorable o desfavorable las cosas buenas / malas le sucedan a la persona en la vida. De modo que, como explica Moyano (2010), la gratitud también permitiría el desarrollo del optimismo en el sentido que ambos rasgos se enfocan en las abstracciones favorables / agradables de la existencia, a saber: la gratitud en el pasado y el presente, y el optimismo disposicional en el presente y futuro; entonces se explicarían las correlaciones inversas entre la gratitud y el pesimismo disposicional.

Se coincide con Moyano (2010) en la idea de configurar el desarrollo de la fortaleza de la gratitud como una herramienta que puede ser empleada en espacios terapéuticos, de orientación / counseling, y por supuesto de psicoeducación. Los resultados expuestos en este trabajo pueden sugerir posibilidades factibles en la promoción de la salud mental, bienestar psicológico y gratitud en contextos educacionales, específicamente en el contexto laboral educativo con docentes a través de la ejecución de intervenciones psicoeducativas eficaces.

De acuerdo a los objetivos de investigación formulados, se pueden enunciar las siguientes conclusiones: a) incremento en las medias del bienestar psicológico y gratitud luego de la aplicación de la intervención, b) aumento estadísticamente significativo en los niveles de bienestar psicológico y fortaleza de la gratitud tomando como referencia las medidas pre y post, y c) correlaciones positivas entre la fortaleza de la gratitud y el bienestar psicológico como inversas entre la gratitud, pesimismo y bienestar psicológico. Por lo tanto, estos hallazgos apoyan los postulados de la psicología positiva orientados a la promoción del bienestar psicológico y salud mental a través de las intervenciones psicológicas específicamente a través de la educación de las fortalezas del carácter; asimismo, son congruentes con la premisa acerca de la relación inversa de las fortalezas del carácter con rasgos / estados / situaciones poco saludables como el pesimismo disposicional. 
A pesar de estos resultados considerados optimistas descritos anteriormente, es importante tomar con cautela los mismos, debido a las limitaciones del estudio, a saber: tamaño pequeño de la muestra, ausencia de grupos controles, asignación no aleatoria de la muestra, todas estas condiciones que pueden afectar en la generalización de los resultados. Sin embargo, desde estas limitaciones se pueden enunciar algunas recomendaciones a modo de líneas futuras de investigación: a) inclusión de grupos controles en el diseño de investigación; b) considerar otras variables dependientes en el diseño de investigación como fortalezas del optimismo y humor, debido a que obedecen también a esquemas cognitivos que podrían ser educados / modificados mediantes psicointervenciones; y c) aplicar el programa de psicología positiva a otros actores educativos inmersos en el contexto educacional: estudiantes, administradores y personal académico.

A pesar de las limitaciones expuestas en este trabajo de investigación se propone que la intervención psicoeducativa descrita cuenta con adecuada evidencia en su efectividad, tomando en consideración que la medición post fue realizada en los meses en los cuales se va cerrando el año escolar, sabiendo que es un periodo de bastante trabajo, ocasional estrés y agotamiento para los docentes (Esteras, Chorot \& Sandín, 2016). A manera de colofón, las evidencias presentadas en este artículo acerca de la efectividad de las intervenciones basadas en psicología positiva en la promoción de la salud mental pueden ser un aliciente para aquellos profesionales centrados en la búsqueda, reivindicación y mantenimiento de la salud ocupacional de los docentes, a pesar y muy pesar de las condiciones laborales del colectivo docente.

\section{Agradecimientos}

Los investigadores agradecen a la Asociación Jóvenes Fuertes Uruguay por el apoyo institucional brindado para el desarrollo del estudio, especialmente a Carina Zerbino e Inés Ruiz. Así como a la institución patrocinadora que seleccionó y financió la intervención psicoeducativa. Y, por supuesto, a todos aquellos docentes participantes del estudio.

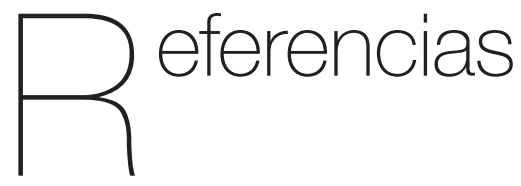

American Psychological Association. (2010). Manual de Publicaciones de la APA. Mexico: Editorial El Manual Moderno

Buschor, C., Proyer, R. T., \& Ruch, W. (2013). Self- and peer-rated character strengths: How do they relate to satisfaction with life and orientations to happiness? Journal of Positive Psychology, 8(2), 116-127

Castro-Solano, A., \& Cosentino, A. C. (2016). The relationships between character strengths and life fulfillment in the view of lay-people in Argentina. Interdisciplinaria Revista de Psicología y Ciencias Afines, 33(1), 65-80.

Casullo, M. (2002). Evaluación del bienestar psicológico en Iberoamérica. Buenos Aires: Paidós. 
Chan, D. W. (2010). Gratitude, gratitude intervention and subjective well-being among Chinese school teachers in Hong Kong. Educational Psychology, 30(2), 139-153.

Chan, D. W. (2011). Burnout and life satisfaction: Does gratitude intervention make a difference among Chinese school teachers in Hong Kong? Educational Psychology, 31(7), 809-823

Chan, D. W. (2013). Counting blessings versus misfortunes: Positive interventions and subjective well-being of Chinese school teachers in Hong Kong. Educational Psychology, 33(4), 504-519

Cunha, L. F., Pellanda, L. C., \& Reppold, C. T. (2019). Positive Psychology and Gratitude Interventions: A Randomized Clinical Trial. Frontiers in Psychology, 10(58), 584-593

Deng, Y., Xiang, R., Zhu, Y., Li, Y., Yu, S., \& Liu, $X$. (2019). Counting blessings and sharing gratitude in a Chinese prisoner sample: Effects of gratitude-based interventions on subjective well-being and aggression. The Journal of Positive Psychology, 14(3), 303-311

Dickens, L. R. (2017). Using gratitude to promote positive change: A series of metaanalyses investigating the effectiveness of gratitude interventions. Basic and Applied Social Psychology, 39(4), 193-208

Ejecutivo, U. P. (2008). Decreto 379/008. Investigación en Seres Humanos. Recuperado de http://www. elderechodigital. com. uy/smu/legisla D, 800379

Emmons, R. A., \& McCullough, M. E. (2003). Counting blessings versus burdens:
Experimental studies of gratitude and subjective well-being. Journal of Personality and Social Psychology, 84(2), 377-389

Esteras, J., Chorot, P. \& Sandín, B. (2016). Un estudio longitudinal de la prevalencia del síndrome de burnout en los docentes según el momento escolar. En J. L. Castejón (Coord.). Psicología y Educación: Presente y Futuro (pp. 1186-1193). Alicante: ACIPE- Asociación Científica de Psicología y Educación.

Ferrando, P. J., Chico, E., \& Tous, J. M. (2002). Propiedades psicométricas del test de optimismo Life Orientation Test. Psicothema, 14(3), 673-680

Giménez, M. (2010). La medida de las fortalezas psicológicas en adolescentes (VIAY): relación con clima familiar, psicopatología y bienestar psicológico (Tesis Doctoral). Universidad Complutense de Madrid, España. Recuperado de https://www.tdx. cat/handle/10803/516293

Hernández, R., Fernández, C. \& Baptista, P. (2008). Metodología de la investigación cientifica. México: Mc Graw Hill.

Jans-Beken, L., Lataster, J., Peels, D. Lechner, L., \& Jacobs, N. (2018). Gratitude, psychopathology and subjective wellbeing: results from a 7.5-month prospective general population study. Journal of Happiness Studies, 19(6), 1673-1689

Lavy, S. (2019). A review of character strengths interventions in twenty-first-century schools: Their importance and how they can be fostered. Applied Research in Quality of Life, 1-24

Martínez-Martí, M. L., Avia, M. D., \& HernándezLloreda, M. J. (2010). The effects of counting 
blessings on subjective well-being: A gratitude intervention in a Spanish sample. The Spanish Journal of Psychology, 13(2), 886-896

Montero, l., \& León, O. G. (2007). A guide for naming research studies in Psychology. International Journal of clinical and Health psychology, 7(3), 847-862.

Moyano, N. C. (2010). Gratitud en la psicología positiva. Psicodebate. Psicología, Cultura y Sociedad, (10), 103-118

Park, N., \& Peterson, C. (2009). Strengths of character in schools. In R. Gilman, E. S. Huebner, \& M. J. Furlong (Eds.), Handbook of positive psychology in schools (pp. 6576). New York: Routledge

Peterson, C., \& Seligman, M. E. (2004). Character strengths and virtues: $A$ handbook and classification. New York, Oxford University Press.

Peterson, C., Ruch, W., Beerman, U., Park, N., \& Seligman, M. E. P. (2007). Strengths of character, orientations to happiness, and life satisfaction. Journal of Positive Psychology, 2, 149-156.

Porto-Noronha, A. P., \& da Fonseca-Martins, D. (2016). Associations between character strengths and life satisfaction: A study with college students. Acta Colombiana de Psicología, 19(2), 97-103.

Proyer, R. T., Ruch, W., \& Buschor, C. (2012). Testing strengths-based interventions: A preliminary study on the effectiveness of a program targeting curiosity, gratitude, hope, humor, and zest for enhancing life satisfaction. Journal of Happiness Studies, 14(1), 275-292
Reivich, K., Gillham, J. E., Chaplin, T. M., \& Seligman, M. E. (2013). From helplessness to optimism: The role of resilience in treating and preventing depression in youth. In Handbook of resilience in children (pp. 201214). Springer, Boston, MA

Rojas, R. \& Feldman, L. (2010). Validación del Inventario de Fortalezas (VIA) en trabajadores venezolanos del sector público. En Memorias del Tercer Foro de las Américas en Factores Psicosociales: Estrés y Trabajo.

Ryff, C. D. (1989). Happiness is everything, or is it? Explorations on the meaning of psychological well-being. Journal of Personality and Social Psychology, 57(6), 1069-1081

Ryff, C. D. (2014). Psychological well-being revisited: Advances in the science and practice of eudaimonia. Psychother Psychosom, 83(1), 10-28.

Scheier, M. F., \& Carver, C. S. (1985). Optimism, coping, and health: assessment and implications of generalized outcome expectancies. Health Psychology, 4(3), 219-247.

Seligman, M. (2011). Flourish: A Visionary New Understanding of Happiness and Wellbeing. Estados Unidos de Norteamérica: Free Press, New York

Sin, N. L., \& Lyubomirsky, S. (2009). Enhancing well-being and alleviating depressive symptoms with positive psychology interventions: A practice-friendly metaanalysis. Journal of Clinical Psychology, 65(5), 467-487.

Vela, J. C., Sparrow, G. S., Ikonomopoulos, J., Gonzalez, S. L., \& Rodriguez, B. (2017). 
The role of character strengths and family importance on Mexican American college students' life satisfaction. Journal of Hispanic Higher Education, 16(3), 273-285.

Watkins, P. C., Under, J., \& Pichinevskiy, S. (2015). Grateful recounting enhances subjective well-being: The importance of grateful processing. The Journal of Positive Psychology, 10(2), 91-98.
Wellenzohn, S., Proyer, R. T., \& Ruch, W. (2016). Humor-based online positive psychology interventions: A randomized placebocontrolled long-term trial. The Journal of Positive Psychology, 11(6), 584-594.

Witvliet, C. V., Richie, F. J., Root Luna, L. M., \& Van Tongeren, D. R. (2019). Gratitude predicts hope and happiness: A two-study assessment of traits and states. The Journal of Positive Psychology, 14(3), 271-282. 